\title{
Two-dimensional Raman spectra of atomic solids and liquids
}

$\operatorname{AUTHOR}(S)$ :

Nagata, Y; Tanimura, Y

\section{CITATION:}

Nagata, Y ...[et al]. Two-dimensional Raman spectra of atomic solids and liquids. JOURNAL OF CHEMICAL PHYSICS 2006, 124(2): 024508.

\section{ISSUE DATE:}

2006-01-14

URL:

http://hdl.handle.net/2433/49827

\section{RIGHT:}

Copyright 2006 American Institute of Physics. This article may be downloaded for personal use only. Any other use requires prior permission of the author and the American Institute of Physics. 


\title{
Two-dimensional Raman spectra of atomic solids and liquids
}

\author{
Yuki Nagata ${ }^{a)}$ and Yoshitaka Tanimura \\ Department of Chemistry, Kyoto University, Oiwakecho, Kitashirakawa, Sakyoku, Kyoto 606-8502, Japan
}

(Received 18 July 2005; accepted 28 September 2005; published online 11 January 2006)

\begin{abstract}
We calculate third- and fifth-order Raman spectra of simple atoms interacting through a soft-core potential by means of molecular-dynamics (MD) simulations. The total polarizability of molecules is treated by the dipole-induced dipole model. Two- and three-body correlation functions of the polarizability at various temperatures are evaluated from equilibrium MD simulations based on a stability matrix formulation. To analyze the processes involved in the spectroscopic measurements, we divide the fifth-order response functions into symmetric and antisymmetric integrated response functions; the symmetric one is written as a simple three-body correlation function, while the antisymmetric one depends on a stability matrix. This analysis leads to a better understanding of the time scales and molecular motions that govern the two-dimensional (2D) signal. The 2D Raman spectra show novel differences between the solid and liquid phases, which are associated with the decay rates of coherent motions. On the other hand, these differences are not observed in the linear Raman spectra. (C) 2006 American Institute of Physics. [DOI: 10.1063/1.2131053]
\end{abstract}

\section{INTRODUCTION}

One important aspect of molecular vibrational spectroscopies is the ability to monitor ultrafast relaxation dynamics controlled by complex inter- and intramolecular interactions. ${ }^{1}$ Vibrational relaxations are in principle dependent upon the configurations of atoms; therefore, we may expect that the information about the local environments of molecules can be obtained by analyzing changes in spectra as functions of conditions such as phase, density, and temperature. However, conventional lincar spectroscopy does not reveal such changes because the large broadening caused by damping and inhomogenity makes spectral peaks featureless. To conquer these difficulties, two-dimensional (2D) vibrational spectroscopies such as fifth-order Raman spectroscopy ${ }^{2-4}$ and third-order infrared (IR) spectroscopy ${ }^{5-9}$ have been proposed. In fifth-order Raman spectroscopy a system is perturbed by two pairs of Raman pulses separated by period $t_{1}$ and then probed after another period $t_{2}$; whereas in third-order IR spectroscopy a system is perturbed by three IR pulses separated by periods $t_{1}$ and $t_{2}$ and then probed after another period $t_{3}$. These $2 \mathrm{D}$ spectroscopies enable us to evaluate the detailed interrogation of the interactions and configurations between molecules, because the contributions to the signals from harmonic vibrational motions vanish in multidimensional spectroscopies due to the fact that the Gaussian integral is involve in three-body correlation function, i.e., $\left\langle\left[\left[q\left(t_{1}+t_{2}\right), q\left(t_{1}\right)\right], q(0)\right]\right\rangle$ for the fifth-order Raman processes, and the destructive interferences between vibrational excitations in four-body correlation function, i.e., $\left\langle\left[\left[\left[q\left(t_{1}+t_{2}+t_{3}\right), q\left(t_{1}+t_{2}\right)\right], q\left(t_{1}\right)\right], q(0)\right]\right\rangle$ for third-order IR spectroscopy. ${ }^{10,11}$ A very large body of theoretical works on 2D Raman and IR spectroscopies have been devoted to the study of inhomogenity, ${ }^{2,12}$ anharmonicity, ${ }^{10,13,14}$ rotational motion, ${ }^{15}$ vibrational dephasing, ${ }^{11,16-20}$ inter- and intramo-

${ }^{a)}$ Electronic mail: nagata@kuchem.kyoto-u.ac.jp lecular interactions, ${ }^{21-23}$ nonlinear system-bath coupling, ${ }^{11,16}$ conformal change, ${ }^{24,25}$ initial condition, ${ }^{26,27}$ phase matching conditions, ${ }^{28}$ and chemical reactions. ${ }^{29}$ Molecular-dynamics (MD) simulations have been performed ranging from liquids $^{30-40}$ to more complex molecules such as peptides. $^{41-43}$

2D Raman spectroscopies are advantageous in studying molecular dynamics in condensed phases because Raman pulses can create instantaneous vibrational excitations on the molecular system and their coherence can be detected by spectroscopic means. ${ }^{44-47}$ The sensitivities of the $2 \mathrm{D}$ spectra to anharmonicity of the potential and the nonlinear dependence of polarizability on nuclear coordinate have been theoretically demonstrated by the use of simple models and have been clarified to some extent with the help of quantum Liouville pathway treatments. ${ }^{48-50}$ But most of these studies have not yet provided helpful pictures of the processes giving rise to particular spectral features in both experimental data $\mathrm{a}^{3,4}$ and MD simulations. ${ }^{30-36}$ Moreover, there has been little guidance from theory on how to distinguish motions of solids and liquids based on these spectroscopies.

In this paper we carry out MD simulations to investigate how the fifth-order response functions measured in 2D Raman spectroscopy depend on temperature and thermodynamic state. We have chosen the soft-core model for these simulations because its scaling property allows us to discuss the phase transition as a function of temperature. ${ }^{51,52}$ Note that the soft-core potential has been used to model metallic glasses with soft modes and has been one of the widely acknowledged models explaining the so-called "boson peak."53 We explore the use of the symmetric and antisymmetric expressions of the integrated 2D Raman response functions to clarify the interpretation of the spectroscopic data. These functions were originally introduced for an easy check of the simulation results. ${ }^{54}$ We then project the $2 \mathrm{D}$ profiles onto two kinds of one-dimensional (1D) maps: one is the fifth-order 
response functions on the $t_{1}=t_{2}$ axis, ${ }^{36}$ and the other is the antisymmetric integrated response function on the $t_{1}=t_{2}$ axis. Because these functions can be constructed from experimental data as well as simulation results, they will be valuable for analyzing the effects of nonlinear dynamics, for instance, resulting from anharmonicity of the potential. The results of our MD simulations indicate that 2D Raman spectroscopy can detect the change in the character of molecular motions in different phases in a way that cannot be observed in thirdorder Raman spectroscopy. On the other hand, the profile of the 2D Raman signal is not sensitive to the temperature changes as long as the system is in the same phase. Moreover, when the symmetric integrated response function, which has the form of a simple three-body correlation function, is compared with the antisymmetric one which has the form of a three-body correlation function including the stability matrix, it is realized what a critical role the stability matrix plays in extracting dynamical information from the fifth-order response function.

In Sec. II, we explain our model and simulation procedures. In Sec. III, we introduce the symmetric and antisymmetric integrated response functions and in Sec. IV we analyze the calculated signal with these functions. In Sec. V, we discuss the temperature and phase effects on the signals. Finally, Sec. VI is devoted to concluding remarks.

\section{COMPUTATIONAL DETAILS OF RESPONSE FUNCTIONS}

We perform microcanonical MD simulations with a periodic boundary condition on a system with 108 spheres interacting via a soft potential, ${ }^{51}$

$$
U(r)=\varepsilon\left(\frac{\sigma}{r}\right)^{6}+A\left(\frac{r}{\sigma}\right)^{4}+B,
$$

where $\varepsilon$ and $\sigma$ are the potential parameters, and the constants $A$ and $B$ are chosen to connect the force and potential smoothly at the cutoff $r_{0}$. Thus $A$ and $B$ are given by $3 / 2 r_{0}^{4}\left[\varepsilon\left(\sigma / r_{0}\right)^{6}\right]$ and $-5 / 2\left[\varepsilon\left(\sigma / r_{0}\right)^{6}\right]$, respectively. The molecular system is controlled by laser pulses. The optical response of the system is then described by a correlation function of the polarizability. The total polarizability is treated using a dipole-induced dipole (DID) model, which can be expressed as ${ }^{55,56}$

$\Pi(t)=\sum_{m}\left(\alpha_{m}-\sum_{n \neq m}\left(\frac{\alpha_{m} \alpha_{n}}{r_{m n}^{3}(t)}-\frac{3\left(\alpha_{m} \cdot \mathbf{r}_{m n}(t)\right)\left(\mathbf{r}_{m n}(t) \cdot \alpha_{n}\right)}{r_{m n}^{5}(t)}\right)\right)$,

where $\alpha_{m}$ is the molecular polarizability of atom $m$. The second term is important because it possesses the information on the configuration of the surrounding particles. The third- and fifth-order response functions, $R_{a b c d}^{(3)}\left(t_{1}\right)$ and $R_{a b c d e f}^{(5)}\left(t_{2}, t_{1}\right)$, which are associated with the 1D and 2D Raman spectroscopies, respectively, are given as follows. ${ }^{2}$

$$
R_{a b c d}^{(3)}\left(t_{1}\right)=\frac{i}{\hbar}\left\langle\left[\Pi_{a b}\left(t_{1}\right), \Pi_{c d}(0)\right]\right\rangle,
$$

$$
R_{a b c d e f}^{(5)}\left(t_{2}, t_{1}\right)=\left(\frac{i}{\hbar}\right)^{2}\left\langle\left[\left[\Pi_{a b}\left(t_{2}\right), \Pi_{c d}(0)\right], \Pi_{e f}\left(-t_{1}\right)\right]\right\rangle,
$$

where $\Pi_{a b}(t)$ is the $a b$ tensor element of the polarizability at time $t,\lfloor\hat{A}, \hat{B}\rfloor \equiv \hat{A} \hat{B}-\hat{B} \hat{A}$ is the quantum commutator, and $\langle\cdots\rangle$ is an ensemble average over an equilibrium initial distribution. To relate these quantum expressions to their classical analogs, it is helpful to rewrite the response functions in terms of the canonical correlation functions. By using Kubo's identity, ${ }^{57}$

$$
\left[e^{-\beta H}, X\right]=e^{-\beta H} \int_{0}^{\beta} d \lambda e^{\lambda H}[X, H] e^{-\lambda H},
$$

where $\beta=1 / k T$. The third- and fifth-order response functions can be written as

$$
R_{a b c d}^{(3)}\left(t_{1}\right)=-\beta\left\langle\dot{\Pi}_{a b}\left(t_{1}\right) ; \Pi_{c d}(0)\right\rangle
$$

and

$$
\begin{aligned}
R_{a b c d e f}^{(5)}\left(t_{2}, t_{1}\right)= & -\beta\left\langle\left[\Pi_{a b}\left(t_{2}\right), \Pi_{c d}(0)\right] ; \dot{\Pi}_{e f}\left(-t_{1}\right)\right\rangle \\
= & \beta^{2}\left\langle\dot{\Pi}_{e f}\left(-t_{1}\right) ; \dot{\Pi}_{c d}(0) ; \Pi_{a b}\left(t_{2}\right)\right\rangle \\
& -\beta\left\langle\left\langle\Pi_{a b}\left(t_{2}\right) ;\left[\Pi_{c d}(0), \Pi_{e f}\left(-t_{1}\right)\right]\right\rangle\right\rangle,
\end{aligned}
$$

where we introduced the canonical correlation functions defined by

$\langle\hat{F} ; \hat{G}\rangle \equiv \frac{\int_{0}^{\beta} d \lambda \operatorname{tr}\left\{e^{-\beta \hat{H}} e^{\lambda \hat{H}} \hat{F} e^{-\lambda \hat{H}} \hat{G}\right\}}{\beta \operatorname{tr}\left\{e^{-\beta \hat{H}}\right\}}$,

$\langle\hat{E} ; \hat{F} ; \hat{G}\rangle \equiv \frac{\int_{0}^{\beta} d \lambda \int_{0}^{\beta} d \lambda^{\prime} \operatorname{tr}\left\{e^{-\beta \hat{H}} e^{\lambda \hat{H}} \hat{E} e^{-\left(\lambda-\lambda^{\prime}\right) \hat{H}} \hat{F} e^{-\lambda^{\prime} \hat{H}} \hat{G}\right\}}{\beta^{2} \operatorname{tr}\left\{e^{-\beta \hat{H}}\right\}}$,

and

$$
\langle\langle\hat{E} ;[\hat{F}, \hat{G}]\rangle\rangle \equiv \frac{\int_{0}^{\beta} d \lambda \operatorname{tr}\left\{e^{-\beta \hat{H}} \hat{E}\left[e^{-\lambda \hat{H}} \hat{F} e^{\lambda \hat{H}}, \hat{G}\right]\right\}}{\beta \operatorname{tr}\left\{e^{-\beta \hat{H}}\right\}} .
$$

In the classical limit, the operators $\hat{E}$ and $\hat{F}$ commute with $e^{\lambda \dot{H}}$ and we have ${ }^{31,54}$

$$
\begin{aligned}
R_{a b c d}^{(3)}\left(t_{1}\right)=- & \beta\left\langle\dot{\Pi}_{a b}\left(t_{1}\right) \Pi_{c d}(0)\right\rangle, \\
R_{a b c d e f}^{(5)}\left(t_{2}, t_{1}\right)= & \beta^{2}\left\langle\Pi_{a b}\left(t_{2}\right) \dot{\Pi}_{c d}(0) \dot{\Pi}_{e f}\left(-t_{1}\right)\right\rangle \\
& -\beta\left\langle\Pi_{a b}\left(t_{2}\right)\left\{\Pi_{c d}(0), \dot{\Pi}_{e f}\left(-t_{1}\right)\right\}_{\mathrm{PB}}\right\rangle \\
= & -\beta\left\langle\left\{\Pi_{a b}\left(t_{2}\right), \Pi_{c d}(0)\right\}_{\mathrm{PB}} \dot{\Pi}_{e f}\left(-t_{1}\right)\right\rangle,
\end{aligned}
$$

where $\{\cdots\}_{\mathrm{PB}}$ denotes the Poisson bracket producing stability 
matrices $\partial p_{k}\left(t_{2}\right) / \partial q_{1}(0)$ representing how large the deviation of the momentum of atom $k$ at time $t_{2}$ is caused by the slight displacement of atom $l$ at time $0 .{ }^{44}$ The significance of the fifth-order measurement arises from the correlation function including the stability matrix, since the stability matrix carries the information on the interference of the particle trajectories that cannot be obtained from the third-order measurement.

There are two approaches to evaluate these signals using MD simulations, the equilibrium approach ${ }^{32,35,36}$ and the nonequilibrium approach. ${ }^{33,36}$ Here we adopt the former because it best reveals the importance of the stability matrix to determine the nature of the fifth-order Raman response. In the equilibrium approach, we first carry out the equilibrium simulation, then evaluate the response function using the trajectories of particles obtained from the simulations.

To carry out the simulation, we set $\varepsilon=1.0, \sigma=1.0$, and $m=1.0$ without loss of generality, where $m$ is the particle mass. Because of the scaling property of the soft-core potential, temperature multiplied by the Boltzmann constant $k T$ is chosen as a parameter with fixed density $\rho=1.0$. We use a fourth-order symplectic integrator method with a time step of 0.01 . The particles form a face-centered cubic (fcc) lattice for $k T \leqslant 0.19$, and they behave like liquid for $0.19 \leqslant k T$. There is also a body-centered cubic (bcc) phase around $k T$ $\approx 0.19$, but the region of this phase is relatively narrow. ${ }^{51,52}$ Here we calculate the third- and fifth-order response functions in the fcc solid and liquid phases. The MD simulations start from a fcc configuration with double the targeted temperature and cool the system by velocity rescaling at the rate of 0.998 for each time step. After the kinetic energy of the system reaches $k T=0.001$, we heat the system up to the targeted temperature. In both cases, we sample over 60000 trajectories by preparing different initial configurations to calculate the response functions, and we use more than 3500 time steps to stabilize the kinetic energy. As the polarizability is independent of momentum, only $\partial p\left(t_{2}\right) / \partial q(0)$ of the stability matrix element is calculated, which decreases to onefourth the computational cost of evaluating the full stability matrices and reduces the memory requirements for sampling trajectories.

\section{FIFTH-ORDER SYMMETRIC AND ANTISYMETRIC INTEGRATED RESPONSE FUNCTIONS}

As has been shown in many previous studies, the 2D profiles of the fifth-order Raman signals are very sensitive to the anharmonicity of the molecular dynamics and nonlinear dependence of the polarizability. But interpreting the 2D profiles in terms of the underlying dynamics is difficult due to the complex connection between the dynamics and spectroscopy. Since the most significant and interesting contribution to the fifth-order signals comes from the term with the stability matrix, ${ }^{36}$ it is valuable and versatile if we can separate it from the others. For this purpose, we utilize the symmetric integrated response function introduced by Cao et al. ${ }^{54}$ and the antisymmetric integrated response function.

To simplify the following explanation, we choose $z$ direction for all tensors and afterward we omit the tensor no- tation. To make the symmetric form with respect to $t_{1}$ and $t_{2}$ from the fifth-order response function, we integrate Eq. (12) with respect to $t_{1}$,

$$
\begin{aligned}
W\left(t_{2}, t_{1}\right) \equiv & \frac{1}{\beta} \int_{0}^{t_{1}} R^{(5)}\left(t_{2}, t^{\prime}\right) d t^{\prime} \\
= & -\beta\left[\left\langle\Pi\left(t_{2}\right) \dot{\Pi}(0) \Pi\left(t_{1}\right)\right\rangle-\left\langle\Pi\left(t_{2}\right) \dot{\Pi}(0) \Pi(0)\right\rangle\right] \\
& +\left\langle\Pi\left(t_{2}\right)\left\{\Pi(0), \Pi\left(-t_{1}\right)\right\}_{\mathrm{PB}}\right\rangle \\
= & \left\langle\left\{\Pi\left(t_{2}\right), \Pi(0)\right\}_{\mathrm{PB}} \Pi\left(-t_{1}\right)\right\rangle \\
& -\left\langle\left\{\Pi\left(t_{2}\right), \Pi(0)\right\}_{\mathrm{PB}} \Pi(0)\right\rangle .
\end{aligned}
$$

Then, by using the relations

$$
\begin{aligned}
\frac{1}{\beta} \int_{0}^{\infty} R^{(5)}\left(t_{2}, t^{\prime}\right) d t^{\prime} & =\beta\left\langle\Pi\left(t_{2}\right) \dot{\Pi}(0) \Pi(0)\right\rangle \\
& =-\left\langle\left\{\Pi\left(t_{2}\right), \Pi(0)\right\}_{\mathrm{PB}} \Pi(0)\right\rangle,
\end{aligned}
$$

and

$$
\left\langle\left\{\Pi\left(t_{1}\right), \Pi(0)\right\}_{\mathrm{PB}} \Pi\left(-t_{2}\right)\right\rangle=\left\langle\Pi\left(t_{2}\right)\left\{\Pi(0), \Pi\left(-t_{1}\right)\right\}_{\mathrm{PB}}\right\rangle,
$$

we have the symmetric integrated response functions as

$$
\begin{aligned}
S\left(t_{2}, t_{1}\right) \equiv & W\left(t_{2}, t_{1}\right)-W\left(t_{1}, t_{2}\right) \\
= & \beta\left\langle\Pi\left(t_{2}\right) \dot{\Pi}(0) \Pi\left(-t_{1}\right)\right\rangle+\beta\left\langle\Pi\left(t_{2}\right) \dot{\Pi}(0) \Pi(0)\right\rangle \\
& -\beta\left\langle\Pi(0) \dot{\Pi}(0) \Pi\left(-t_{1}\right)\right\rangle .
\end{aligned}
$$

Here, we also introduce its counterpart, the antisymmetric integrated response function,

$$
\begin{aligned}
A\left(t_{2}, t_{1}\right) \equiv & W\left(t_{2}, t_{1}\right)+W\left(t_{1}, t_{2}\right) \\
= & \left\langle\Pi\left(t_{2}\right)\left\{\Pi(0), \Pi\left(-t_{1}\right)\right\}_{\mathrm{PB}}\right\rangle \\
& -\left\langle\Pi(0)\left\{\Pi(0), \Pi\left(-t_{1}\right)\right\}_{\mathrm{PB}}\right\rangle \\
& +\left\langle\left\{\Pi\left(t_{2}\right), \Pi(0)\right\}_{\mathrm{PB}} \Pi\left(-t_{1}\right)\right\rangle \\
& -\left\langle\left\{\Pi\left(t_{2}\right), \Pi(0)\right\}_{\mathrm{PB}} \Pi(0)\right\rangle
\end{aligned}
$$

Although no special attention has previously been paid to the antisymmetric integrated response function, we find that it contains the key to analyzing liquid dynamics because it isolates the contribution of the stability matrix from that of the simple three-body correlation function. In the following, we demonstrate this point by using the present model in the solid and liquid phases. If the above expressions are described with the normal mode, the last two terms in Eqs. (16) and (17) negate with the terms such as $\Pi^{\prime} \Pi^{\prime} \Pi$ [see Eq. (23)]. Thus, the leading terms of the symmetric and antisymmetric integrated response functions both involve $\Pi^{\prime \prime} \Pi^{\prime} \Pi^{\prime}$.

As is evident from the definition, these functions satisfy the relations,

$$
S\left(t_{2}, t_{1}\right)=-S\left(t_{1}, t_{2}\right)
$$

and

$$
A\left(t_{2}, t_{1}\right)=A\left(t_{1}, t_{2}\right)
$$

Moreover, the functions are orthogonal to one another, 


$$
\iint d t_{1} d t_{2} A\left(t_{2}, t_{1}\right) \cdot S\left(t_{2}, t_{1}\right)=0
$$

which indicates that the information of the symmetric integrated response functions is independent to that of the antisymmetric ones.

The symmetric integrated response function $S\left(t_{2}, t_{1}\right)$ is useful for self-consistent checks of numerical simulations, ${ }^{54}$ because it does not involve the stability matrix and its numerical calculation is $1 / N^{2}$ times faster than that of the full fifth-order response function. On the contrary, calculation of the antisymmetric integrated response function $A\left(t_{2}, t_{1}\right)$ requires the same cost as calculation of the fifth-order response function. But it contains the important information on coherent molecular motions described by the stability matrix. It is of importance to notice that although the present analysis is based on the MD simulation, one can also construct the symmetric and antisymmetric integrated response functions from experimental data. By utilizing these functions, one can quantitatively discuss the effects of nonlinear dynamics, for instance, from anharmonicity.

To illustrate the nature of these functions, we calculate them from the soft-core model in the solid and liquid phases. In Fig. 1, we plot the fifth-order response function $R^{(5)}\left(t_{2}, t_{1}\right)$, the symmetric and antisymmetric integrated response functions, $S\left(t_{2}, t_{1}\right)$ and $A\left(t_{2}, t_{1}\right)$, respectively, and the derivatives of $S\left(t_{2}, t_{1}\right)$ and $A\left(t_{2}, t_{1}\right)$ with respect to $t_{1}$,

$$
R_{S}^{(5)}\left(t_{2}, t_{1}\right) \equiv \partial S\left(t_{2}, t_{1}\right) / \partial t_{1}
$$

and

$$
R_{A}^{(5)}\left(t_{2}, t_{1}\right) \equiv \partial A\left(t_{2}, t_{1}\right) / \partial t_{1},
$$

for $k T=0.155$ in the solid phase. To compare the contributions of the symmetric and antisymmetric components of the fifth-order response function, in Fig. 2 we plot the diagonal slices $R^{(5)}(t, t), R_{S}^{(5)}(t, t) \equiv \partial S\left(t_{2}, t_{1}\right) /\left.\partial t_{1}\right|_{t=t_{1}=t_{2}}$, and $R_{A}^{(5)}(t, t)$ $\equiv \partial A\left(t_{2}, t_{1}\right) /\left.\partial t_{1}\right|_{t=t_{1}=t_{2}} \quad$ of $\quad R^{(5)}\left(t_{2}, t_{1}\right), \quad R_{S}^{(5)}\left(t_{2}, t_{1}\right), \quad$ and $R_{A}^{(5)}\left(t_{2}, t_{1}\right)$. Notice that $R^{(5)}\left(t_{2}, t_{1}\right)=R_{S}^{(5)}\left(t_{2}, t_{1}\right)+R_{A}^{(5)}\left(t_{2}, t_{1}\right)$.

The position of the peak appearing in $R^{(5)}\left(t_{2}, t_{1}\right)$ is not necessarily located on the $t_{1}=t_{2}$ axis. ${ }^{39}$ In fact, we can estimate that the peak position in Fig. 1(a) is $\left(t_{2}, t_{1}\right)$ $=(0.77,0.64)$ by using a parabolic interpolation. Analyzing the fifth-order signal is difficult because 2D profiles of the signals are usually featureless and the locations of their peaks do not necessarily correspond to specific physical processes. Although the profiles can be changed with the physical conditions, it is hard to choose which portions of the 2D signals to make comparisons. Since the antisymmetric integrated response function always exhibits a symmetric peak along $t_{1}=t_{2}$ axis and is sensitive to the physical conditions due to the contribution from the stability matrix term, it is more instructive to analyze the signals using it instead of the fifth-order response function. In the following, we discuss the parameter dependences of the 2D Raman signals with the help of the antisymmetric integrated response function.
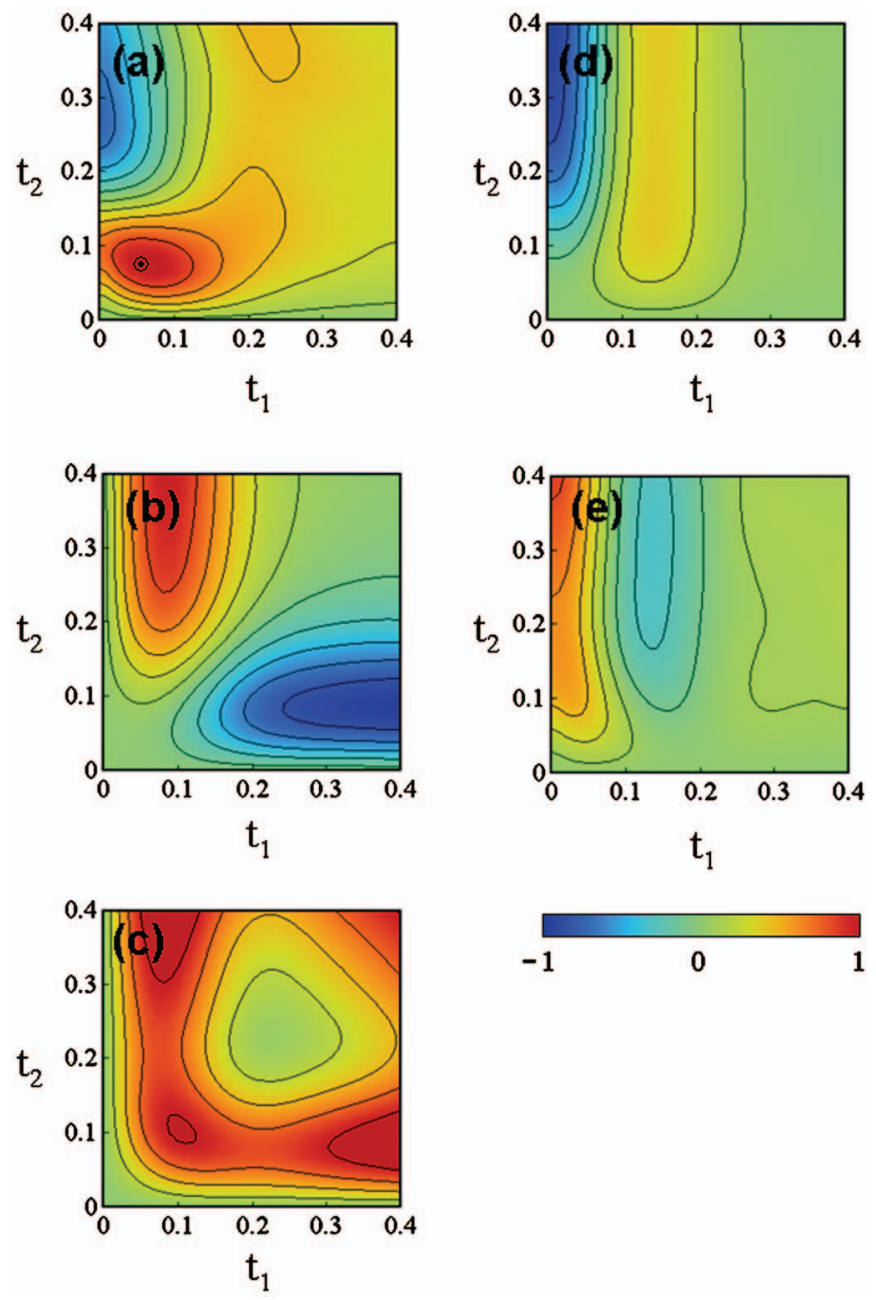

$-1$

1

FIG. 1. (Color) (a) $R^{(5)}\left(t_{2}, t_{1}\right)$, (b) $S\left(t_{2}, t_{1}\right)$, (c) $A\left(t_{2}, t_{1}\right)$, (d) $R_{S}^{(5)}\left(t_{2}, t_{1}\right)$, and (e) $R_{A}^{(5)}\left(t_{2}, t_{1}\right)$ at $k T=0.155$ in the solid phase. The peak position at $\left(t_{2}, t_{1}\right)$ $=(0.77,0.64)$ is denoted by a double circle in Fig. 1(a).

\section{SYMMETRIC AND ANTISYMMETRIC INTEGRATED RESPONSE FUNCTIONS FOR NORMAL-MODE ANALYSIS}

In a normal-mode analysis, molecular motions are represented by an ensemble of oscillatory motions along the nuclear coordinates. ${ }^{2,30,31,48,54,58}$ This analysis is often useful since it gives us access to the microscopic dynamics related to a specific vibrational frequency. ${ }^{59}$ Since the fifth-order experiments have the ability to measure anharmonic vibrational motions through the stability matrix term, it is interesting to observe how the symmetric and antisymmetric integrated response functions contribute to the signals in the normalmode analysis.

When the polarizability is given in powers of a molecular coordinate as

$$
\begin{aligned}
\Pi(t)= & \Pi(0)+\Pi^{\prime}(q(t)-q(0)) \\
& +\frac{1}{2} \Pi^{\prime \prime}(q(t)-q(0))^{2}+\cdots,
\end{aligned}
$$

the third- and fifth-order response functions $R^{(3)}\left(t_{1}\right)$ and $R^{(5)}\left(t_{2}, t_{1}\right)$ can be expressed as ${ }^{2}$

$$
R^{(3)}\left(t_{1}\right)=\left\langle\Pi^{\prime} \Pi^{\prime} C^{\prime \prime}\left(t_{1}\right)\right\rangle \propto \int d \omega \rho(\omega) \frac{\sin \left(\omega t_{1}\right)}{\omega},
$$




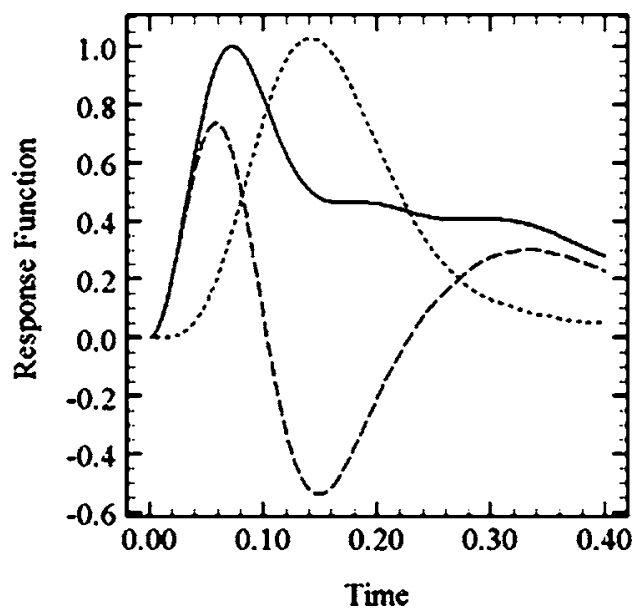

FIG. 2. Diagonal slices $\left(t=t_{1}=t_{2}\right)$ from the signals depicted in Fig. 1: $R^{(5)}(t, t)$ (solid curve), $R_{S}^{(5)}(t, t)$ (dotted), and $R_{A}^{(5)}(t, t)$ (dashed).

$$
\begin{aligned}
R^{(5)}\left(t_{2}, t_{1}\right) & =\left\langle\Pi^{\prime \prime} \Pi^{\prime} \Pi^{\prime} C^{\prime \prime}\left(t_{2}\right)\left(C^{\prime \prime}\left(t_{1}\right)+C^{\prime \prime}\left(t_{1}+t_{2}\right)\right)\right\rangle \\
& \propto \int d \omega \rho(\omega) \frac{\sin \left(\omega t_{2}\right)\left(\sin \left(\omega t_{1}\right)+\sin \left(\omega\left(t_{1}+t_{2}\right)\right)\right)}{\omega^{2}},
\end{aligned}
$$

where $C^{\prime \prime}(t)=\sin (\omega t) / \omega$. We then obtain the following results on $t=t_{1}=t_{2}$,

$$
\begin{aligned}
& S(t, t)=0, \\
& A(t, t) \propto \int d \omega \rho(\omega) \frac{\sin ^{3}(\omega t)}{\omega^{3}} .
\end{aligned}
$$

Since $S(t, t)$ gives zero, we substitute $R_{S}^{(5)}(t, t)$ and $R_{A}^{(5)}(t, t)$ for $S(t, t)$ and $A(t, t)$,

$$
\begin{aligned}
& R_{S}^{(5)}(t, t) \propto \int d \omega \rho(\omega) \frac{\sin ^{2}(\omega t)(1-\cos (\omega t))}{\omega^{2}}, \\
& R_{A}^{(5)}(t, t) \propto \int d \omega \rho(\omega) \frac{\sin ^{2}(\omega t) \cos (\omega t)}{\omega^{2}} .
\end{aligned}
$$

$R^{(5)}(t, t), R_{S}^{(5)}(t, t)$, and $R_{A}^{(5)}(t, t)$ can be expressed in terms of $R^{(3)}(t)$ as

$$
\begin{aligned}
& R^{(5)}(t, t) \propto \int_{0}^{t} d t\left(6 R^{(3)}(3 t)+4 R^{(3)}(2 t)-2 R^{(3)}(t)\right), \\
& R_{S}^{(5)}(t, t) \propto \int_{0}^{t} d t\left(-3 R^{(3)}(3 t)+4 R^{(3)}(2 t)+R^{(3)}(t)\right), \\
& R_{A}^{(5)}(t, t) \propto \int_{0}^{t} d t\left(9 R^{(3)}(3 t)-3 R^{(3)}(t)\right) .
\end{aligned}
$$

Thus, by comparing $R_{S}^{(5)}(t, t)$ and $R_{A}^{(5)}(t, t)$ calculated from the fifth-order response functions with $\int_{0}^{t} d t\left(-3 R^{(3)}(3 t)\right.$ $\left.+4 R^{(3)}(2 t)+R^{(3)}(t)\right)$ and $\int_{0}^{t} d t\left(9 R^{(3)}(3 t)-3 R^{(3)}(t)\right)$, we can easily estimate the contribution from the anharmonic dynamical terms resulting from the stability matrix and the validity of the normal-mode expressions. In Fig. 3, we compare $R^{(5)}(t, t), R_{S}^{(5)}(t, t)$, and $R_{A}^{(5)}(t, t)$ with their expressions in terms of $R^{(3)}(t)$ under the normal-mode assumption. Figure 3(a) clearly shows us that the normal-mode expressions fail to predict the fifth-order response functions even in the shorttime region. However, if we focus on Fig. 3(b), we see that the normal-mode expressions for the symmetric integrated response functions are valid in the region $t \leqslant 0.10$. The normal-mode analysis can describe the accurate dynamics in the short time, ${ }^{58}$ which is consistent with the results of the symmetric integrated response functions. One of the reasons for the large deviation at $t \geqslant 0.10$ can be deduced from Brownian motion theory. When the function $C^{\prime \prime}(t)$ is expressed by

$$
C^{\prime \prime}(t)=\frac{1}{\sqrt{\omega^{2}-\zeta^{2} / 4}} e^{-\zeta t / 2} \sin \left(\sqrt{\omega^{2}-\zeta^{2} / 4} t\right)
$$

in the Brownian oscillator model, ${ }^{2}$ the quantities calculated from the fifth-order response function decay more rapidly than the predictions using the third-order response function. On the other hand, in Fig. 3(c), $R_{A}^{(5)}(t, t)$ deviates from $\int_{0}^{t} d t\left(9 R^{(3)}(3 t)-3 R^{(3)}(t)\right)$ even for $t \leqslant 0.10$, which indicates that the anharmonic contribution from the stability matrix $\left\langle\Pi^{\prime} \Pi^{\prime} \Pi^{\prime}\left(\partial q\left(t_{2}\right) / \partial p(0)\right) \dot{q}\left(-t_{1}\right)\right\rangle$ plays an important part in the antisymmetric integrated response function. Moreover, the deviation of the fifth-order response function from the normal-mode expression shown in Fig. 3(a) is mainly caused by the antisymmetric part.

\section{TEMPERATURE DEPENDENCE}

We discuss the response functions in the solid and liquid phases at different temperatures. In Fig. 4(a), we depict the third-order response functions $R^{(3)}(t)$ for $k T=0.215$ and 0.20 in the liquid phases and for $k T=0.155$ and 0.14 in the solid phases. $^{60}$

The profiles of the third-order signals in Fig. 4(a) are similar but their decay times are slightly different for different temperatures. Under three approximations, the Gaussian decaying correlation, the lattice gas model, and the WeeksChandler-Anderson scheme, the third-order response function in the short-time region is expressed as ${ }^{61}$

$$
R^{(3)}(t) \propto \frac{t}{\tau^{2}} \exp \left(-\frac{t^{2}}{\tau^{2}}\right),
$$

where $\tau$, the decay time, relates to temperature of the system via

$$
\tau \propto T^{-1 / 2}
$$

Equation (34) indicates that the positions of the first peaks in third-order response functions are proportional to $T^{-1 / 2}$. In Fig. 4(b) we plot the peak positions obtained from the simulations as a function of temperature. The peak positions are dependent on temperature and if the phases are different, the gradients are different. The calculation of the gradients of the peak positions as a function of temperature, however, requires high-accuracy measurements that it seems to be difficult to observe the difference from experimental means.

Now we discuss the fifth-order signals. The fifth-order response functions $R^{(5)}\left(t_{2}, t_{1}\right)$ are shown in Fig. 5. To illustrate the difference between solid and liquid phases, we also 

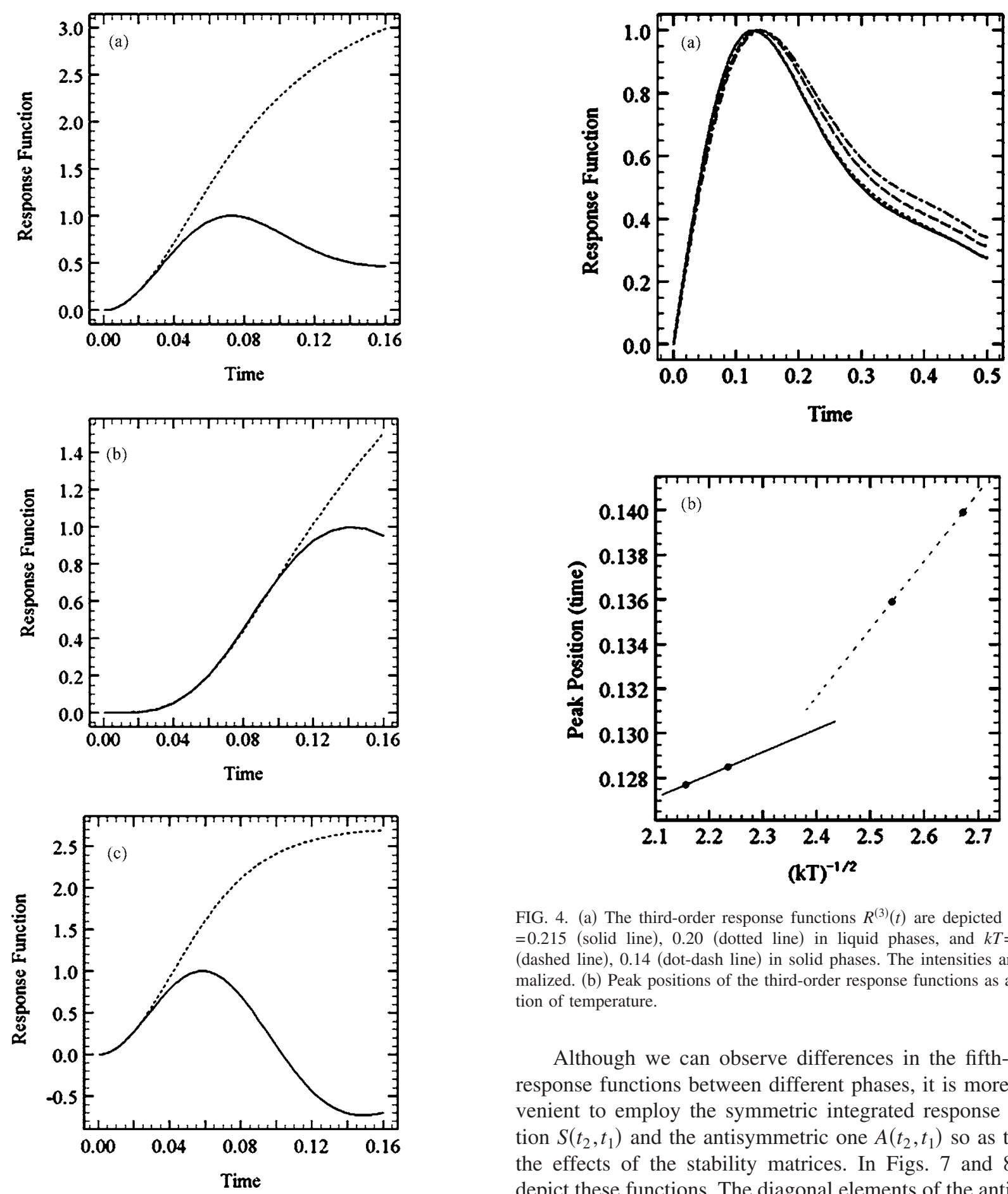

FIG. 3. (a) $R^{(5)}(t, t)$ (solid line) and $\int_{0}^{t} d t\left(6 R^{(3)}(3 t)+4 R^{(3)}(2 t)-2 R^{(3)}(t)\right)($ dotted line), (b) $R_{S}^{(5)}(t, t)$ (solid line) and $\int_{0}^{t} d t\left(-3 R^{(3)}(3 t)+4 R^{(3)}(2 t)+R^{(3)}(t)\right)$ (dotted line), and (c) $R_{A}^{(5)}(t, t)$ (solid line) and $\int_{0}^{t} d t\left(9 R^{(3)}(3 t)-3 R^{(3)}(t)\right)$ (dotted line) at $k T=0.155$ in the solid phase. In each figure, the intensities of the solid lines are normalized at the first peak and the dotted lines are scaled so that their derivatives are same as those of solid lines near $t=0$.

plot $R^{(5)}(t, t)$ for different temperatures as 1D maps in Fig. 6 . There are differences between Figs. 5(a) and 5(b) in the liquid phases and Figs. 5(c) and 5(d) in the solid phases for $t_{1} \geqslant 0.2$ and $t_{2} \geqslant 0.2$. Figure 6 clearly shows that the phase change leads to a change of the spectral decay rates especially for $t \geqslant 0.15$, while the temperature change has a limited effect as long as the system is in the same phase.

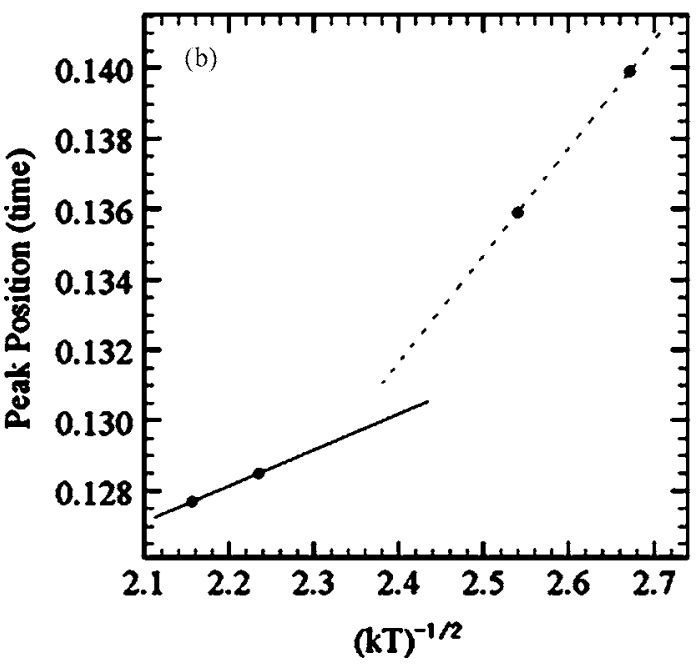

FIG. 4. (a) The third-order response functions $R^{(3)}(t)$ are depicted for $k T$ $=0.215$ (solid line), 0.20 (dotted line) in liquid phases, and $k T=0.155$ (dashed line), 0.14 (dot-dash line) in solid phases. The intensities are normalized. (b) Peak positions of the third-order response functions as a function of temperature.

Although we can observe differences in the fifth-order response functions between different phases, it is more convenient to employ the symmetric integrated response function $S\left(t_{2}, t_{1}\right)$ and the antisymmetric one $A\left(t_{2}, t_{1}\right)$ so as to see the effects of the stability matrices. In Figs. 7 and 8, we depict these functions. The diagonal elements of the antisymmetric integrated response functions $A(t, t)$ are also plotted in Fig. 9. While we observe similar tendencies among the results of $S\left(t_{2}, t_{1}\right)$ in Figs. 7(a)-7(d), we see the clear difference among the results of $A\left(t_{2}, t_{1}\right)$ in the liquid and solid phases in Figs. $8(\mathrm{a})-8(\mathrm{~d})$ in $t_{1} \geqslant 0.2$ and $t_{2} \geqslant 0.2$ and Fig. 9 in $t$ $\geqslant 0.15$. Although we cannot do longer simulations due to the limitations to our computational power, we may expect the bigger and clearer differences in the decay rates in the antisymmetric response functions to appear in the longer-time region. The sensitivity of $A\left(t_{2}, t_{1}\right)$ and insensitivity of $S\left(t_{2}, t_{1}\right)$ to the phase change result from the stability matrix which reveals deviations from harmonic motion as the interference between the trajectories. The antisymmetric integrated response functions, which can be obtained from experimental 


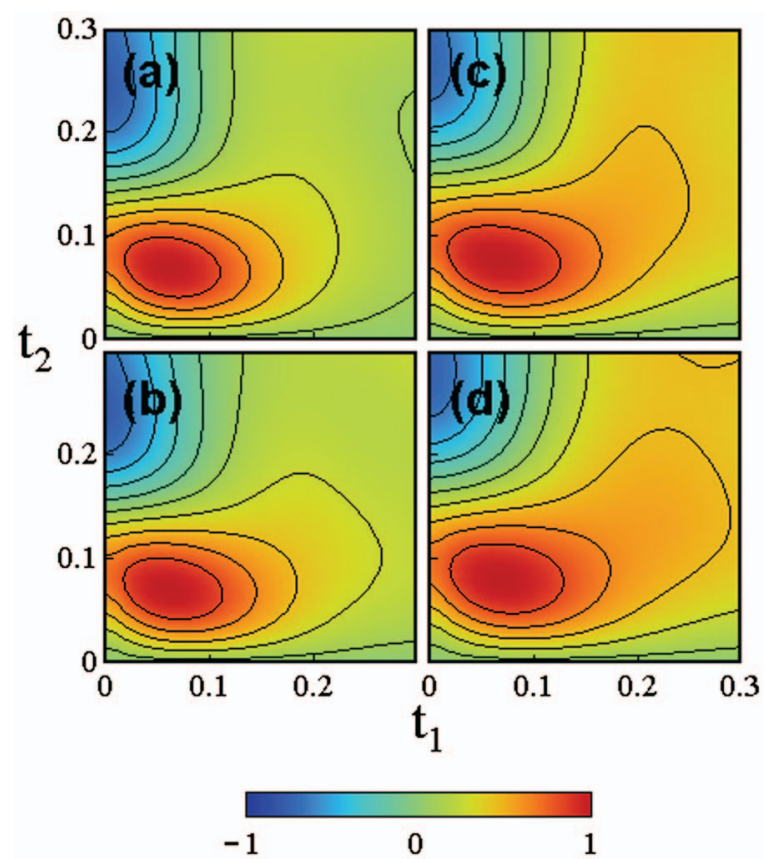

FIG. 5. (Color) The classical fifth-order response functions $R^{(5)}\left(t_{2}, t_{1}\right)$ for the cases (a) $k T=0.215$, (b) $k T=0.20$ in the liquid phase and (c) $k T=0.155$, (d) $k T=0.14$ in the solid phase. The intensity of each plot is normalized at the first peak.

data as well as simulation results by following the procedure explained in Sec. III, are more valuable than the symmetric ones, because the former allow us to estimate the contribution from the stability matrix directly.

\section{CONCLUSIONS}

Using the MD simulation, we calculated the third- and fifth-order Raman signals of atomic solids and liquids described by the soft-core potential. To analyze these signals quantitatively and to reveal the effect of the stability matrix, we have decomposed the fifth-order response function into the symmetric and antisymmetric integrated response func-

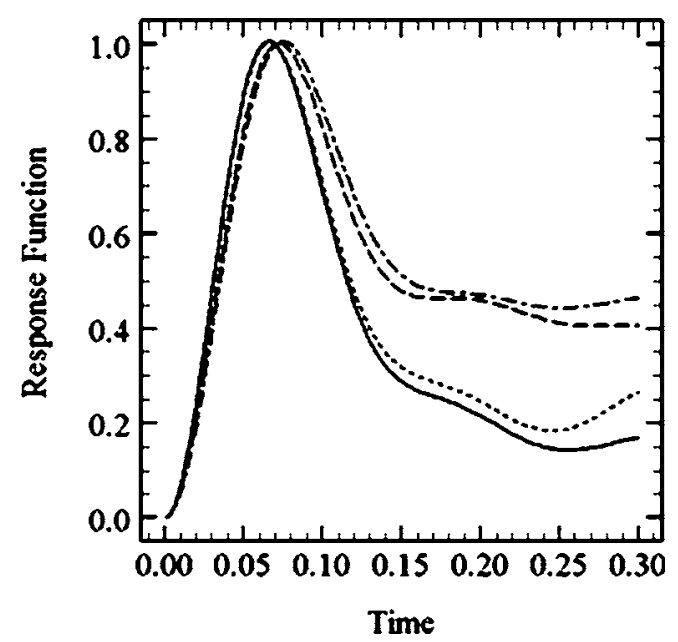

FIG. 6. The diagonal elements $R^{(5)}(t, t)$ are illustrated for $k T=0.215$ (solid line), 0.20 (dotted line) in the liquid phase and $k T=0.155$ (dashed line), 0.14 (dot-dash line) in the solid phase. The intensity of each plot is normalized at the first peak.

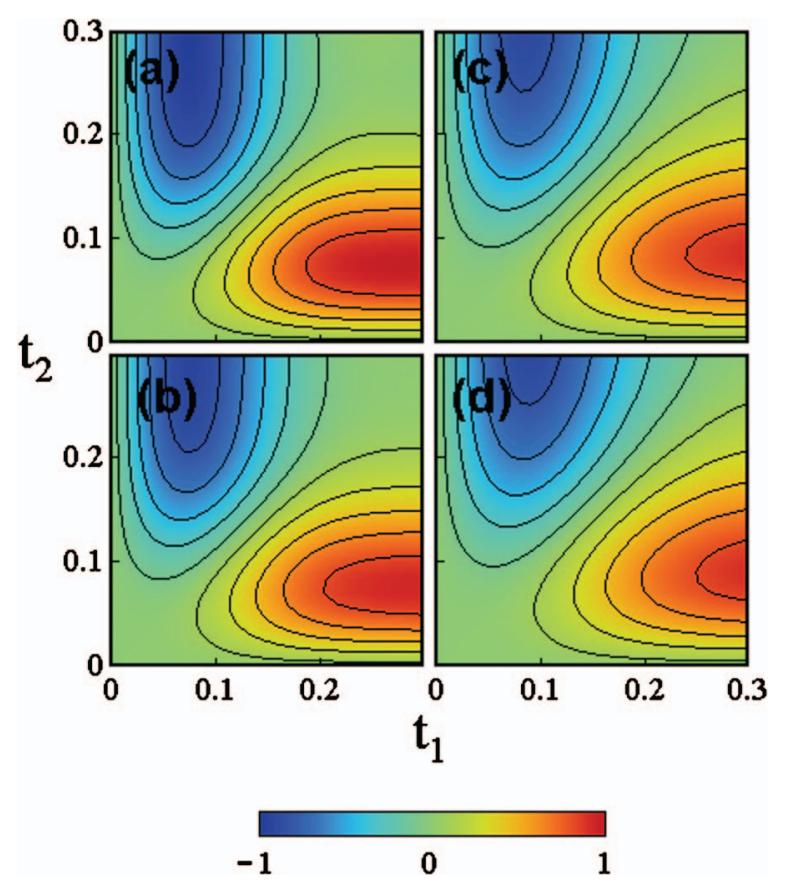

FIG. 7. (Color) The symmetric integrated response functions $S\left(t_{2}, t_{1}\right)$ are plotted as two-dimensional contour maps for the cases (a) at $k T=0.215$ and (b) at $k T=0.20$ in liquid phase and (c) at $k T=0.155$ and (d) at $k T=0.14$ in solid phase. The intensity of each figure is normalized at the first peak.

tions; the symmetric one has the form of the simple threebody correlation function, while the antisymmetric one also includes contributions from the stability matrix. The latter cannot be expressed accurately in terms of normal-mode expressions even in the short-time region, where the symmetric integrated response function can be. This fact indicates that

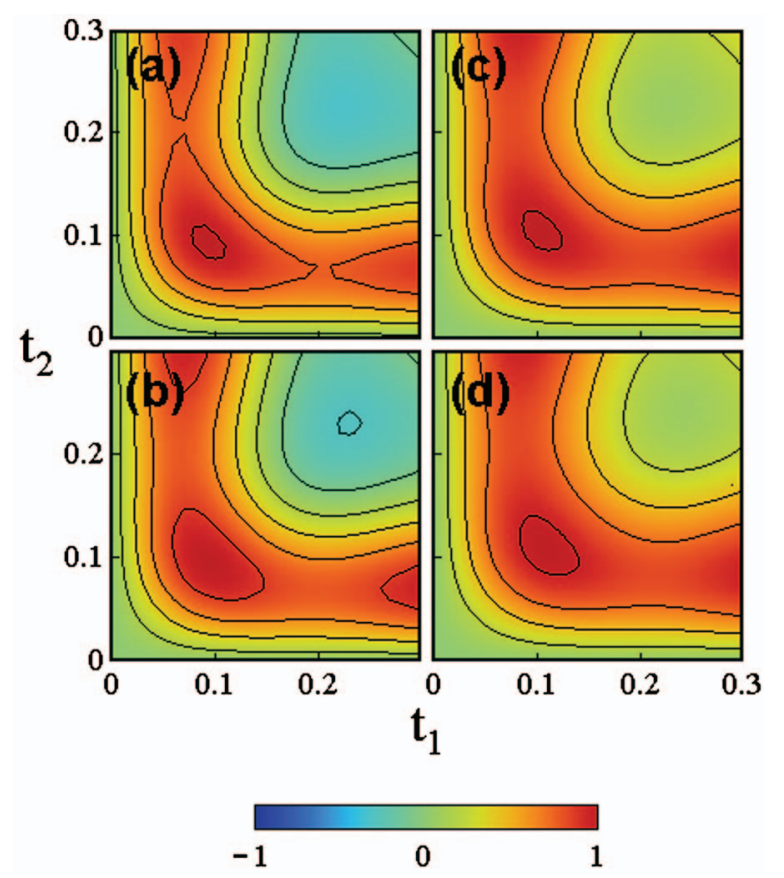

FIG. 8. (Color) The differences of the antisymmetric integrated response functions $A\left(t_{2}, t_{1}\right)$ are plotted as two-dimensional contour maps for the cases (a) at $k T=0.215$ and (b) at $k T=0.20$ in liquid phase and (c) at $k T=0.155$ and (d) at $k T=0.14$ in solid phase. The intensity of each plot is normalized at the first peak. 


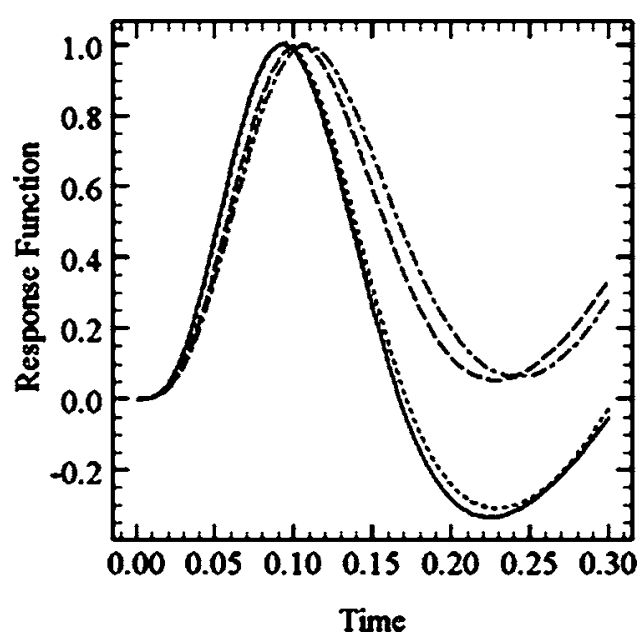

FIG. 9. The diagonal elements $A(t, t)$ are illustrated for $k T=0.215$ (solid line), 0.20 (dotted line) in liquid phase and $k T=0.155$ (dashed line), 0.14 (dot-dash line) in solid phase. The intensity of each plot is normalized at the first peak.

the anharmonic contributions which are missing in the normal-mode expressions give rise to an important effect on the fifth-order signals.

It is shown that the change between liquid and solid phases causes a minor effect on the third-order signals, while it causes dramatic differences in the fifth-order signals. The antisymmetric integrated response functions show prominent changes as a result of the phase transition, whereas the change in the symmetric one is modest. The difference between the symmetric and antisymmetric integrated response functions results from the sensitivity of the stability matrix to the nonlinear dynamics, because the stability matrix reveals the deviation from the harmonic motion as the interference between the trajectories. This result suggests the advantage of using the antisymmetric integrated response functions rather than the fifth-order response functions for analysis. Such features are, however, unable to be revealed within the frame work of the normal-mode analysis, since it neglects the effects from the anharmonicity described by the stability matrix.

Since we can always construct the symmetric and antisymmetric integrated response functions not only from the simulation results but also from the experimental data, these functions will be valuable and versatile tools for analyzing the effects of nonlinear dynamics upon the fifth-order signals.

\section{ACKNOWLEDGMENTS}

We wish to express our gratitude to Professor Shinji Saito and Professor Mark Maroncelli for fruitful discussions. One of the authors (Y.T.) is thankful for the financial support from a Grant-in-Aid for Scientific Research A 15205005 from Japan Society for the Promotion of Science and Morino Science Foundation. Some parts of the numerical calculations were performed at the Computer Center of the Institute for Molecular Science.

${ }^{1}$ S. Mukamel, Principles of Nonlinear Optical Spectroscopy (Oxford University Press, New York, 1995).
${ }^{2}$ Y. Tanimura and S. Mukamel, J. Chem. Phys. 99, 9496 (1993).

${ }^{3}$ V. Astinov, K. J. Kubarych, C. J. Milne, and R. J. D. Miller, Chem. Phys. Lett. 327, 34 (2000); K. J. Kubarych, C. L. Milne, S. Lin, V. Astinov, and R. J. D. Miller, J. Chem. Phys. 116, 2016 (2002).

${ }^{4}$ L. J. Kaufman, J. Heo, L. D. Ziegler, and G. R. Fleming, Phys. Rev. Lett. 88, 207402 (2002).

${ }^{5}$ P. Hamm, M. Lim, and R. M. Hochstrasser, J. Phys. Chem. B 102, 6123 (1998); M. T. Zanni, S. Gnanakaran, J. Stenger, and R. M. Hochstrasser, J. Phys. Chem. B 105, 6520 (2001); S. Gnanakaran and R. M. Hochstrasser, J. Am. Chem. Soc. 123, 12886 (2001).

${ }^{6}$ C. Scheurer, A. Piryatinski, and S. Mukamel, J. Am. Chem. Soc. 123, 3114 (2001)

${ }^{7}$ M. Khalil, N. Demirdoven, and A. Tokmakoff, J. Phys. Chem. A 107, 5258 (2003); C. J. Fecko, J. D. Eaves, J. Loparo, A. Tokmakoff, and P. L. Geissler, Science 301, 1698 (2003).

${ }^{8}$ K. Ohta, H. Maekawa, S. Saito, and K. Tominaga, J. Phys. Chem. A 107, 5643 (2003).

${ }^{9}$ J. B. Asbury, T. Steinel, C. Stromberg, S. A. Corcelli, C. P. Lawrence, J. L. Skinner, and M. D. Fayer, J. Phys. Chem. A 108, 1107 (2004).

${ }^{10}$ K. Okumura and Y. Tanimura, J. Chem. Phys. 106, 1687 (1997); K. Okumura and Y. Tanimura, ibid. 107, 2267 (1997).

${ }^{11}$ T. Steffen and Y. Tanimura, J. Phys. Soc. Jpn. 69, 3115 (2000); Y. Tanimura and T. Steffen, ibid. 69, 4095 (2000).

${ }^{12}$ K. Okumura and Y. Tanimura, Chem. Phys. Lett. 277, 159 (1997).

${ }^{13}$ Y. Tanimura, Chem. Phys. 233, 217 (1998).

${ }^{14}$ R. B. Williams and R. F. Loring, J. Chem. Phys. 113, 1932 (2000)

${ }^{15}$ Y. Suzuki and Y. Tanimura, J. Chem. Phys. 119, 1650 (2003).

${ }^{16}$ T. Kato and Y. Tanimura, J. Chem. Phys. 117, 6221 (2002); J. Chem. Phys. 120, 260 (2004).

${ }^{17}$ J. Sung, R. J. Silbey, and M. Cho, J. Chem. Phys. 115, 1422 (2001).

${ }^{18}$ K. F. Everitt and J. L. Skinner, Chem. Phys. 266, 197 (2001); K. F. Everitt, E. Geva, and J. L. Skinner, J. Chem. Phys. 114, 1326 (2001).

${ }^{19}$ W. G. Noid, G. S. Ezra, and R. F. Loring, J. Phys. Chem. B 108, 6536 (2004); W. G. Noid, G. S. Ezra, and R. F. Loring, J. Chem. Phys. 120, 1491 (2004)

${ }^{20}$ S. Yang, J. Shao, and J. Cao, J. Chem. Phys. 121, 11260 (2004).

${ }^{21}$ K. Okumura and Y. Tanimura, Chem. Phys. Lett. 278, 175 (1997).

${ }^{22}$ A. Tokmakoff, M. J. Lang, D. S. Larsen, G. R. Fleming, V. Chernyak, and S. Mukamel, Phys. Rev. Lett. 79, 2702 (1997).

${ }^{23}$ K. Okumura, D. M. Jonas, and Y. Tanimura, Chem. Phys. 266, 237 (2001).

${ }^{24}$ K. Okumura, A. Tokmakoff, and Y. Tanimura, J. Chem. Phys. 111, 492 (1999).

${ }^{25}$ S. Hahn, K. Kwak, and M. Cho, J. Chem. Phys. 112, 4553 (2000).

${ }^{26}$ Y. Suzuki and Y. Tanimura, J. Chem. Phys. 115, 2267 (2001).

${ }^{27}$ D. F. Underwood and D. A. Blank, J. Phys. Chem. A 109, 3295 (2005); S. J. Schmidtke, D. F. Underwood, and D. A. Blank, J. Am. Chem. Soc. 126, 8620 (2004).

${ }^{28}$ T. Kato and Y. Tanimura, Chem. Phys. Lett. 341, 329 (2001).

${ }^{29}$ O. Kühn and Y. Tanimura, J. Chem. Phys. 119, 2155 (2003); T. Brixner, J. Stenger, H. M. Vaswani, M. Cho, R. E. Blankenship, and G. R. Fleming, Nature (London) 434, 625 (2005).

${ }^{30}$ S. Ryu and R. M. Stratt, J. Phys. Chem. B 108, 6782 (2004).

${ }^{31}$ S. Saito and I. Ohminc, J. Chem. Phys. 108, 240 (1998).

${ }^{32}$ A. Ma and R. M. Stratt, Phys. Rev. Lett. 85, 1004 (2000); A. Ma and R. M. Stratt, J. Chem. Phys. 116, 4972 (2002).

${ }^{33}$ T. I. C. Jansen, J. G. Snijders, and K. Duppen, J. Chem. Phys. 113, 307 (2000); T. I. C. Jansen, J. G. Snijders, and K. Duppen, ibid. 114, 10910 (2001).

${ }^{34}$ R. DeVane, C. Ridley, B. Space, and T. Keyes, J. Chem. Phys. 119, 6073 (2003).

${ }^{35}$ S. Saito and I. Ohmine, Phys. Rev. Lett. 88, 207401 (2002).

${ }^{36}$ S. Saito and I. Ohmine, J. Chem. Phys. 119, 9073 (2003).

${ }^{37}$ J. Cao, J. Wu, and S. Yang, J. Chem. Phys. 116, 3739 (2002).

${ }^{38}$ T. Keyes and J. T. Fourkas, J. Chem. Phys. 112, 287 (2000); J. Kim and T. Keyes, Phys. Rev. E 65, 061102 (2002).

${ }^{39}$ R. A. Denny and D. R. Reichman, J. Chem. Phys. 116, 1987 (2002).

${ }^{40}$ A. Piryatinski and J. L. Skinner, J. Phys. Chem. B 106, 8055 (2002); C. P. Lawrence and J. L. Skinner, J. Chem. Phys. 119, 3840 (2003).

${ }^{41}$ K. Kwac, H. Lee, and M. Cho, J. Chem. Phys. 120, 1477 (2004); H. Torii, Chem. Phys. Lett. 414, 417 (2005).

${ }^{42}$ K. A. Merchant, W. G. Noid, D. E. Thompson, R. Akiyama, R. F. Loring, and M. D. Fayer, J. Phys. Chem. B 107, 4 (2003).

${ }^{43}$ A. M. Moran, S.-M. Park, and S. Mukamel, J. Chem. Phys. 118, 9971 
(2003); T. Hayashi and S. Mukamel, J. Phys. Chem. A 107, 9113 (2003).

${ }^{44}$ S. Mukamel, V. Khidekel, and V. Chernyak, Phys. Rev. E 53, R1 (1996).

${ }^{45}$ M. Cho, in Advances in Multi-photon Process and Spectroscopy, edited by S. H. Lin, A. A. Villaeys, and Y. Fujimura (World Scientific, Singapore, 1999), Vol. 12, p. 229

${ }^{46}$ S. Mukamel, Annu. Rev. Phys. Chem. 51, 691 (2000).

${ }^{47}$ J. T. Fourkas, Adv. Chem. Phys. 117, 235 (2001).

${ }^{48}$ T. Steffen, J. T. Fourkas, and K. Duppen, J. Chem. Phys. 105, 7364 (1996).

${ }^{49}$ K. Okumura and Y. Tanimura, J. Phys. Chem. A 107, 8092 (2003).

${ }^{50}$ W. G. Noid and R. F. Loring, J. Chem. Phys. 121, 7057 (2004).

${ }^{51}$ W. G. Hoover, S. G. Gray, and K. W. Johnson, J. Chem. Phys. 55, 1128 (1971); W. G. Hoover, D. A. Young, and R. Grover, J. Chem. Phys. 56, 2207 (1972)

${ }^{52}$ B. B. Laird and A. D. J. Haymet, Mol. Phys. 75, 71 (1992).

${ }^{53}$ H. R. Schober and B. B. Laird, Phys. Rev. B 44, 6746 (1991).

${ }^{54}$ J. Cao, S. Yang, and J. Wu, J. Chem. Phys. 116, 3760 (2002).
${ }^{55}$ P. A. Madden and D. J. Tildesley, Mol. Phys. 55, 969 (1985).

${ }^{56}$ B. M. Ladanyi, Chem. Phys. Lett. 121, 351 (1985).

${ }^{57}$ R. Kubo, M. Toda, and N. Hashitsume, Statistical Physics II (Springer, New York, 1991).

${ }^{58}$ M. Buchner, B. M. Ladanyi, and R. M. Stratt, J. Chem. Phys. 97, 8522 (1992); R. M. Stratt and M. H. Cho, ibid. 100, 6700 (1994); R. M. Stratt, Acc. Chem. Res. 28, 201 (1995).

${ }^{59}$ M. Cho, G. R. Fleming, S. Saito, I. Ohmine, and R. M. Stratt, J. Chem. Phys. 100, 6672 (1994).

${ }^{60}$ Since we consider a small system of 108 particles in the present simulations, there are large fluctuations of temperature. These fluctuations cause the difficulty in carrying out the simulation near the phase-transition temperature $k T=0.19$ because during the simulation the molecular states may take both solid and liquid phases due to the fluctuation of the temperature. Therefore, we avoid the regions at near $k T=0.19$ in our simulations.

${ }^{61}$ K. Okumura, B. Bagchi, and Y. Tanimura, Bull. Chem. Soc. Jpn. 73, 873 (2000). 